\title{
À la mémoire d'Ellen Gee 29 janvier 1950 au 3 novembre 2002
}

\author{
Susan A. McDaniel \\ Université de l'Alberta
}

Dans son livre intitulé Composing a Life, Mary Catherine Bateson, fille de Margaret Mead et de Gregory Bateson, nous propose que «... les parties les plus intéressantes des histoires de la vie sont celles des échos d'une vie à une autre» (1990: 16). La vie d'Ellen Gee résonnait de vies des autres: celles qu'elle aimait, celles qu'elle enseignait et guidant, celles avec lesquelles elle oeuvrait et celles qu'elle documentait avec respect, soin et reconnaissance.

En plus d'être une collègue sociologue et parfois une collaboratrice, Ellen était mon amie, ma très chère amie. Ses amis et collègues se retrouvent inconsolables suivant sa mort subite en novembre 2002. Son absence résonne. Elle était notre modèle et sa contribution à l'étude d'autrui est incomparable. La cruauté de l'ironie ne passe pas inaperçu. Ellen effectuait des recherches sur le vieillissement, surtout sur le vécu féminin; malheureusement, elle n'aura jamais la chance de vieillir. Elle voyait le potentiel dans ce dernier stage de la vie et souvent, elle affirmait ne jamais vouloir se retirer de sa profession; son vœu s'est cruellement réalisé.

Lors de notre dernière rencontre à Montréal en octobre, elle anticipait un voyage du temps des fêtes avec sa famille à Cuba. Elle y avait déjà voyagé et même si parfois elle travaillait de longues heures, elle appréciait soigneusement le temps passé en famille avec Gordon, son époux, Adrienne, sa fille et Margaret, sa mère. Son dernier courriel était intitulé «Esprit interne» et elle me décrivait sa théorie qui voulait que les chiens aient développé la méthode de détente ultime! Son humour était très contagieux. Elle adorait son chien Sades. Sades lui faisait découvrir des parcs qu'elle ne connaissait pas et la capacité de détente de Sades inspirait Ellen (nous aussi d'ailleurs nous devrions en tirer leçon).

Ellen est née à Vancouver, pays des lotus. Son réseau étendu d'amis, de famille et de collègues servait en sorte de trampoline pour son travail érudit et de lien à la communauté, la politique, les individus et le monde. À l'exception d'un court séjour dans les années 1970 à l'Université de Victoria, sa carrière et sa vie se retrouvaient à Vancouver. Les fameux restaurants et ses Grizzlies bien-aimés ajoutaient à sa vie

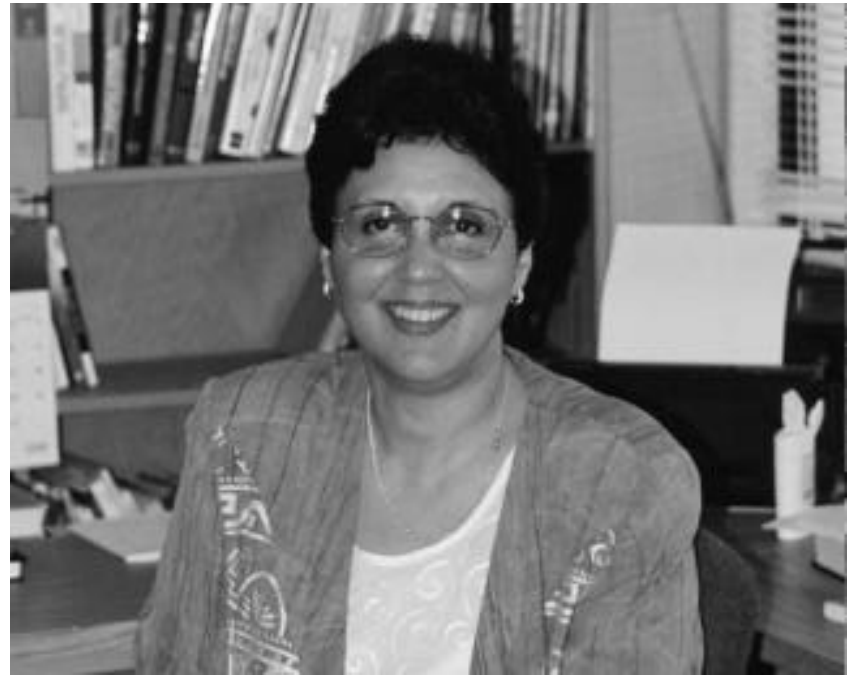

bien remplie. Rien ne la rendait plus heureuse qu'un bon repas en compagnie d'amis, de famille et de collègues. Sa toxicomanie du sushi était légendaire et elle était fanatique des Grizzlies !

Ellen Gee était reconnue comme une érudite et depuis 1994, la Présidente du Département de sociologie et d'anthropologie de l'Université Simon Fraser. Elle a contribué à plusieurs revues savantes et elle a oeuvré comme rédactrice-gestionnaire de la Revue canadienne de sociologie et d'anthropologie et comme rédactrice des sciences sociales de la Revue canadienne du vieillissement. Un mois avant sa mort, elle m'annonçait qu'elle venait d'être invitée à se joindre au Comité de rédaction du Journal of Marriage and Family; ce dernier poste était pour elle un honneur incomparable.

Étant donné ses réussites fécondes, il est difficile d'énumérer ses contributions. Sa co-rédaction avec Gloria Gutman du livre The Overselling of Population Aging: Apocalyptic Demography, Intergenerational Challenges and Social Policy (2000) a certainement incité la curiosité des sciences de la gérontologie, de la démographie et de la politique sociale au Canada et à l'étranger. Ce livre est le résultat de la Conférence John K. Friesen de 1998 (organisée par le Centre de recherche en gérontologie de la SFU). L'idée principale du livre propose que le vieillissement de la population est un signe positif de réussite sociale plutôt qu'une défaite. Les deux auteures ont été invitées à 
présenter une communication sur cette perspective de recherche à Tokyo et juillet 2002, lors d'un forum sur le vieillissement de la population. Ellen et moi-même avions l'intention de rapprocher nos chapitres individuels du livre afin de développer une communication conjointe qui proposerait de dénouer le vieillissement économique du vieillissement démographique. Je travaille seule à cette tâche et cette dernière sera dédiée à la mémoire d'Ellen.

Récemment, Ellen contribua quatre chapitres au livre de Neena Chappell, Ellen Gee, Lynn McDonald et Michael Stones intitulé Aging in Contemporary Canada (2003). Malheureusement, elle ne verra pas l'impression de ce livre. Pendant la production, elle apprenait que le Conseil de recherche en sciences humaines du Canada - Initiative de la nouvelle économie, avait approuvé le financement de l'étude «Workforce Agin in the New Economy: A comparative Study of Information Technology Employment», une bourse de $2,9 \$$ millions. Ellen devait en participer avec Julie McMillun (Université de Western Ontario), chercheuse principale. Je suis membre du Comité consultatif international du projet. Ellen m'a fait part de son enthousiasme lors de son voyage à Londres pour une session de brainstorming traitant du développement de ce projet au stage de la demande. Elle avait hâte d'entreprendre la recherche - le lendemain suivant l'affectation de la bourse, elle se croyait déjà en retard dans ce travail!

Sa recherche sur la série d'études de la vie intergénérationnelle avec Barbara Mitchell et Andrew Wister la passionnait. J'ai toujours pensée que cette recherche était d'une valeur personnelle pour elle, car elle entretenait des relations inter-générationnelles avec Adrienne, sa fille et ses parents. Même si je n'ai jamais connu son père, décédé il y a à peine deux ans, je la voyais souvent avec sa mère et sa fille et j'admirais l'amitié profonde, le respect, l'humour et la joie évidents dans leurs relations. Je lui ai souvent fait part de mes observations en lui disant qu'elle avait de la chance. Elle me répondant, «je le sais» - pas surprenant étant donné sa capacité d'observer les relations interpersonnelles. Ellen était la sociologue quintessencielle, ce qui lui permettait un aperçu unique, combiné à ses recherches sur la vie inter-générationnelle. Les relations inter-générationnelles sont diverses, complexes et offrent un vrai défi. Une communication à ce sujet par Ellen Gee et Barbara Mitchell, «Exploring multi-generational families in Canada», fera l'objet d'un chapitre de Voices: Essays on Canadian Families (2e édition), ce qui sera dirigé par Marion Lynn.

Ellen s'intéressait aussi aux dimensions variées du multiculturalisme, surtout la relation entre ce dernier et le vieillissement ou le bien-être. Lors de son décès, elle oeuvrait avec sa collège Parin Dossa, à une étude financée par CRSHC sur le croisement de la politique et de la santé des immigrantes âgées. En 2002, elle termina avec Ray Corrado, une étude financé par CRSHC sur les représailles des aborigènes. De plus, elle travaillait avec Barbara Mitchell et Andrew Wister sur un projet de la culture et la co-résidence. Elle avait aussi reçu avec Jon Kesselman et d'autres chercheurs, une subvention MCRI de 1,7\$ million traitait du changement de la répartition du bien-être au Canada. Cette dernière recherche se concentrait sur l'étude approfondie de l'ethnicité.

Il n'est pas surprenant d'apprendre qu'Ellen a reçu la distinction du Prix de contribution exceptionnelle de ses collègues sociologues et anthropologues canadiens lors de la Conférence tenue à Québec en 2001. En tant que Présidente élue de la SCSA, j'ai eu le privilège de lui remettre ce prix. Sa mère et sa fille y étaient et je garde en souvenir une photo de nous; Ellen était si belle et pleine de joie.

Pas surprenant non plus, qu'Ellen trouvait le temps d'occuper le poste de Présidente de son département et ce pour les dernières neuf années-assurément un record canadien à battre ! Elle a occupé le poste de Doyenne-adjointe de SFU, à la Faculté des arts de 1988 à 1992 et de Doyenne par intérim de la Faculté des études supérieures en 1992. Elle était membre fondateur du Comité directeur de la gérontologie de SFI dès 1982; elle occupa le poste de Présidente au sein de ce dernier dès 1988.

Il est possible qu'Ellen soit le résultat du premier clonage, car une seule personne ne peut avoir réalisé autant de projets. Toutefois, elle trouvait le temps d'en faire davantage ! Elle était l'amie et le guide de plusieurs. Jane Pulkingham, une collègue, $\mathrm{m}^{\prime}$ a fait parvenir cette pensée suivant le décès d'Ellen: " sa cordialité, sa nature compatissante, son intégrité, son dévouement, son humour ' sel de la terre,' son irrévérence envers les autres et son aptitude à répondre aux besoins des autres, tous cela faisait d'Ellen une amie et collègue très chère.» Karen Kobayashi, ancienne étudiante au doctorat décrit Ellen comme étant «la personne qui fournissait les bons outils à tous et chacun afin que ces derniers réussissent dans leur domaine choisi et dans leur vie. Elle nous a donné un fondement de base sur lequel nous pouvions poursuivre notre carrière érudite et elle nous a enseigné à devenir de bons citoyens du monde.»

La mort subite d'Ellen Gee à l'âge de 52 ans est une perte énorme pour les domaines de la sociologie et de la gérontologie canadienne. Ceux qui la connaissaient ont eu la chance que les histoires de nos vies soient celles des échos de la sienne. Nos vies ont été enrichies davantage par sa présence. «Lorsque l'on 
joue au tennis, et le vent souffre de notre dos, on ne s'en rend pas compte et on pense peut être que l'on joue bien ... ce n'est que lorsque l'on change de court, et le vent se retrouve contre nous que l'on apprécie la force du vent». Nous avons changé de court et reconnaissons la force de ta brise. Merci Ellen.
*Rose, P. (1984). Parallel Lives. New York: Vintage Books, p. 268.

Également publié dans la revue Canadian Journal of Sociology Online January-February 2003. 